\title{
Comparison of Inclusive Charm and Beauty Cross Sections in Deep-inelastic Scattering at HERA with Theoretical Predictions
}

\author{
P. D. Thompson ${ }^{a}$ \\ ${ }^{a}$ E-mail: pdt@ hep.ph.bham.ac.uk, School of Physics, University of Birmingham, \\ Birmingham B15 2TT, UK
}

\begin{abstract}
The measurements of inclusive charm and beauty cross sections in deep-inelastic scattering $e p$ collisions at HERA are compared with the predictions of perturbative quantum chromodynamics from the CTEQ and MRST fitting groups, employing a range of theoretical schemes. The differences in the theoretical predictions are discussed and the theoretical uncertainties investigated.
\end{abstract}




\section{Introduction}

The study of heavy flavour production in deep-inelastic scattering (DIS) $e p$ collisions at HERA provides a test of perturbative quantum chromodynamics (pQCD). In particular, the presence of the heavy quark mass $M$ provides an additional 'hard' scale to the momentum transfer of the exchanged boson $Q$. The perturbative series has to be treated in different ways depending on the relative magnitude of $M$ and $Q$ and is, therefore, a stringent test of the QCD factorization theorem. The description of heavy flavour processes is particularly important in precise global QCD analyses of DIS structure functions as heavy flavours form an increasingly significant contribution to the total cross section with increasing energy. For example, at values of $Q^{2}=$ $650 \mathrm{GeV}^{2}$ charm (beauty) production has been measured [1] to be $\sim 25 \%(\sim 2.5 \%)$ of the total DIS cross section. The measurement of the charm and beauty structure functions, $F_{2}^{c \bar{c}}$ and $F_{2}^{b \bar{b}}$, provides a more direct sensitivity to the gluon distribution of the proton than inclusive structure function $F_{2}$. The understanding of the gluon and quark distributions in the region of low $x$ has important implications for the measurement of standard model and new physics processes at hadron colliders such as the Tevatron and LHC. Recent measurements of $F_{2}^{c \bar{c}}$ and $F_{2}^{b \bar{b}}$ have been made at HERA $[1,2]$ based on a technique using the displacement of tracks from the primary interaction vertex, which arise from long lived charm and beauty hadrons, and have small extrapolations to the inclusive phase space. The measurements of $F_{2}^{c \bar{c}}$ using this technique are found to be compatible with those obtained using a largely independent method based on the reconstruction of exclusive charm mesons [3-6], giving increasing confidence that the extrapolations from the exclusive to the inclusive phase space, for the $Q^{2}$ range of the displaced track measurements, are well controlled.

In this paper the status of the theoretical description of the latest published data and the theoretical uncertainties are evaluated. Firstly, the various theoretical treatments available for the description of heavy flavour production within pQCD are briefly introduced. Secondly, a comparison of the different theoretical schemes with the experimental data is made for $F_{2}^{c \bar{c}}$ and $F_{2}^{b \bar{b}}$. Thirdly, the uncertainties of the theoretical predictions for charm and beauty are compared. In the last section the influence of the gluon distribution on the cross sections is investigated in more detail. These investigations aim to provide insight into the areas of phase space where future measurements of heavy flavour data can contribute to best improve the theoretical understanding.

\section{Theoretical Treatments of Heavy Flavour Production}

In this section the three sets of the most common theoretical treatments of heavy quark production in DIS are briefly introduced. The first two methods attempt to reduce the dual scale problem involving $Q$ and $M$ to an effective, and hence approximate, single scale problem. The third scheme tries to unify the two different approaches, which are valid at the extremes of phase space, to provide the most accurate description over the whole kinematic range. The sets of parton distribution functions (PDFs) considered in this paper, which employ all three heavy flavour schemes, are restricted to those from the two QCD fitting groups of CTEQ $[7,8]$ and MRST [9-11]. 


\subsection{Fixed Flavour Number Scheme}

In order to correctly describe the production of a pair of heavy flavour quarks around the threshold of $W=2 M$, where $W$ is the photon-proton centre of mass energy, the mass of the heavy quark should be explicitely taken into account in the calculations. This is done in the fixed flavour number scheme (FFNS) where the heavy flavour quarks are treated as 'heavy' particles, never as massless partons. The leading order production process for heavy flavour in this scheme is based on the 'massive' boson-gluon fusion (BGF) matrix element to order $\alpha_{s}$, generated mainly from the gluon distribution of the proton. The BGF process is one order higher in $\alpha_{s}$ than the lowest order quark parton model (QPM) contribution for the production of light quarks. The maximum number of flavours $N_{f}$ contributing to the strong coupling evolution is limited to the maximum number of 'light' or massless flavours. In the case where both charm and beauty are treated as massive $N_{f}=3$. In the case where charm is treated as massless and beauty as massive then $N_{f}=4$. In this paper only those FFNS PDFs for which $N_{f}=3$ are considered. The heavy flavour FFNS BGF-like coefficients have been calculated to next-to-leading order (NLO) i.e. to order $\alpha_{s}^{2}$ [12].

The FFNS provides the most reliable description of heavy flavour production around the kinematic threshold 11 (in the absence of a non-perturbative contribution). However, the presence of mass dependent terms of the form $\ln Q^{2} / M^{2}$, which are kept in the expansion for all values of $Q^{2}$, spoils the convergence of the series as $Q^{2} \rightarrow \infty$. QCD does not predict the scale at which the terms become infra-red unsafe and, as will be seen, the FFNS provides an adequate description of the existing experimental HERA data over the entire kinematical range. However, theoretically it remains prudent to take these divergent terms into account. This is dealt with by the variable flavour number schemes described in the next sections. Another disadvantage of the FFNS is that the hard-scattering matrix elements are generally an order of $\alpha_{s}$ higher than the 'massless' calculations and have not been calculated for many processes, for example, charged current scattering. This is particularly problematic for global QCD analyses where many of the standard data sets which are used in the fits have to be omitted in a FFNS fit, resulting in less well constrained partons.

The FFNS is implemented by the CTEQ and MRST groups for either three (CTEQ5F3 [7], MRST2004FF3 [11]) or four (CTEQ5F4 [7], MRST2004FF4 [11]) flavours of light quarks. The limited applicability of the FFNS is the reason for the absence of FFNS PDFs in the most recent set of CTEQ parton densities (CTEQ6.5 [8]) i.e. there are no CTEQ6F3 or CTEQ6F4 PDFs. However, parton sets compatible with the FFNS are still essential for making predictions for the hadronic final state in ep collisions since the main NLO program HVQDIS [13], which implements the massive BGF matrix elements to NLO and provides differential cross sections for the heavy quarks, is calculated in this scheme. The MRST2004FF partons have been produced specially for this purpose from another set of PDFs which used a more general scheme for heavy flavour production (see section 2.3). It should be noted that because the MRST2004FF PDFs are not extracted from a FFNS fit themselves, they generally provide a poor description of the inclusive proton structure function $F_{2}$.

As was highlighted in [11] the original calculations of the massive NLO coefficient functions were evaluated for the fixed flavour definition of the strong coupling $\alpha_{s}^{N_{f}=3,4}$. Therefore, when

\footnotetext{
${ }^{1}$ The threshold of $W=2 M$ corresponds to $Q^{2}=4 M^{2} x /(1-x)$.
} 
fitting at NLO in the FFNS a compatible fixed flavour definition of $\alpha_{s}$ should be used. For the H1 [14] and ZEUS [15] FFNS fits the more commonly used variable five flavour definition of $\alpha_{s}^{N_{f}=5}$ was used. The incompatibility of coefficients and schemes leads to too much evolution at low $Q^{2}$ and too little evolution at higher $Q^{2}$, causing inaccuracies for the predictions of heavy flavour cross sections. In order to use a value of $\alpha_{s}^{N_{f}=3,4}$ compatible with the world average value of $\alpha_{s}^{N_{f}=5}\left(M_{Z}\right) \sim 0.118$ in a FFNS fit, the couplings (via the value of $\Lambda^{Q C D}$ ) should be matched at some low scale (typically $m_{c}$ ), so that it is possible to describe the bulk of the experimental data that are in the low $Q^{2}$ region. Note that when the fixed three-flavour definition of $\alpha_{s}^{N_{f}=3,4}$ is evolved to $M_{Z}$ it will have a low value $\alpha_{s}\left(M_{Z}\right) \sim 0.1$ when compared with the world average. The correct FFNS implementation was used by $\mathrm{H} 1$ in a recent fit to inclusive diffractive data [16]. The ZEUS collaboration use the Thorne-Roberts scheme [17](see section 2.3) to account for heavy flavour mass effects in their standard PDFs $[15,18]$.

\subsection{Zero Mass Variable Flavour Number Scheme}

The simplest way to resum the divergent $\ln Q^{2} / M^{2}$ terms in the perturbative expansion is to absorb them into the parton distribution functions by treating them as massless partons in the standard way. In the zero mass-variable flavour number scheme (ZM-VFNS) the heavy flavour particles are treated as massless partons. The heavy flavour partons contribute to the cross section and evolution of $\alpha_{s}$ only when the factorization scale $\mu_{f}$ is larger than some threshold value, usually chosen as $M$. Since in the ZM-VFNS $Q$ is often used as the factorization scale, the heavy flavour parton distributions are zero below $Q^{2}<M^{2}$ and all other parton distributions are continuous across the threshold $Q^{2}=M^{2}$. In the ZM-VFNS the heavy flavour parton densities provide the correct theoretical behaviour when $Q^{2}$ is large $\left(Q^{2} \gg M^{2}\right)$ but the approximation $M=0$ deteriorates as $Q^{2}$ becomes the same order of magnitude as $M^{2}$. To improve the applicability of the ZM-VFNS at low $Q^{2}$ more general schemes have been developed and are introduced below.

\subsection{General Mass Variable Flavour Number Schemes}

Whilst neither the FFNS nor the ZM-VFNS can provide individually a satisfactory theoretical description of heavy flavour production over the whole kinematic range, the most reliable predictions can be obtained by combining the two and utilising the most appropriate scheme at a particular $Q^{2}$. The first scheme to attempt to unify the perturbative reliability of the ZM-VFNS at large $Q^{2}$, whilst introducing a finite quark mass around threshold to correctly describe the production of heavy flavours at low $Q^{2}$, was developed by the ACOT [19] group of collaborators. In simple terminology, the DIS cross section in the VFNS 3 may be considered as the sum of the fixed flavour and massless terms and a term which represents the overlap between the two, which needs to be subtracted in order to avoid double counting of the cross section.

\footnotetext{
${ }^{2}$ This holds to NLO but at NNLO the heavy flavour parton distributions may be none zero below $Q^{2}=M^{2}$ and the other parton distributions may be discontinuous across the mass thresholds.

${ }^{3}$ The formalism is often also referred to as the general mass (GM) VFNS to help distinguish it from the ZMVFNS.
} 
Although all theoretical groups adopt many of the underlying ideas of the ACOT scheme there remains some freedom in the prescription for treating the scattering terms in different orders of $\alpha_{s}$ across the heavy flavour mass thresholds.

The most recent set of standard PDFs from CTEQ (CTEQ6.5) is based on an implementation of the general mass ACOT scheme that incorporates the $\operatorname{ACOT}(\chi)$ [20] rescaling variable for a more accurate treatment of the kinematics, the S-ACOT [21] prescription for a simplified treatment of the Wilson coefficients and other features, as described in [8].

The MRST group adopt the Thorne-Roberts (TR) [17] prescription for the VFNS. The TR VFNS is based on the ACOT scheme and implements the same heavy quark coefficient function choice of the ACOT $(\chi)$ prescription for $F_{2}^{c \bar{c}}$ and $F_{2}^{b \bar{b}}$ but differs in other choices. This scheme is used in the standard MRST2004 set of parton distributions. The TR VFNS was recently extended to NNLO [10]. As the order $\alpha_{s}^{3}$ scattering coefficients for massive heavy flavour production have yet to be calculated, approximations, that were found to be successful in estimating NNLO effects in the inclusive cross section, are made. For this paper an update of the NNLO partons compared to those originally appearing in [10] are used.

As discussed in section 2.1 the most recent heavy flavour ep final state calculation program available HVQDIS still uses the FFNS and, therefore, comparison with the results from the latest VFNS PDFs from CTEQ and MRST (with the exception of the specially generated MRST2004FF sets) is difficult.

\subsection{Summary of PDFs and Parameters}

In this section the main QCD schemes for heavy flavour production have been introduced. The PDFs from CTEQ and MRST which are used in this paper are summarised in table 1 . As with all QCD calculations there are a number of choices in the parameters and scales used. The table shows the values used in the original extraction of the PDFs by the fitting groups. The effect of the variations of these parameters is investigated in section 4.

The masses of the heavy quarks are important in the predictions and are shown in table 1 The values for CTEQ and MRST are $5-10 \%$ different. The quark masses in the table are the 'on-shell' mass or pole mass [22] and are, therefore, slightly larger than the latest values in the $\overline{M S}$ scheme from the PDG [22] of $\bar{m}_{c}=1.25 \pm 0.09 \mathrm{GeV}$ and $\bar{m}_{b}=4.20 \pm 0.07 \mathrm{GeV}$. In comparisons of theoretical predictions with final state measurements the 'on-shell' mass values and variations typically used are $m_{c}=1.5 \pm 0.2 \mathrm{GeV}$ and $m_{b}=4.75 \pm 0.25 \mathrm{GeV}$ [23].

Other important parameters for heavy flavour production are the factorization $\mu_{f}$ and renormalization $\mu_{r}$ scales. The former sets the scale at which the parton distribution is sampled and the latter the scale at which $\alpha_{s}$ is evaluated. In the case of light quark production $\mu_{f}$ and $\mu_{r}$ are usually set to $Q$. The presence of a 'heavy' quark creates an additional uncertainty in the choice of the perturbative scale. The perturbative scale in the expansion of the heavy flavour coefficients $\mu_{M}$ may be different to that chosen for the light quarks. For the MRST PDFs $\mu_{M}$ is chosen to be the same as for the light quarks $\mu_{M}=Q$. For the most recent PDF from CTEQ (CTEQ6.5) the scale for heavy flavour is chosen as $\mu_{M}=\sqrt{Q^{2}+M^{2}}$. As discussed in [8] the heavy flavour structure functions $F_{2}^{c \bar{c}}$ and $F_{2}^{b \bar{b}}$ are theoretically not infra-red safe beyond NLO. 
However, the effects are expected to be small and comparison with experimental results is still possible, although the NLO predictions may be sensitive to the choice of parameters such as $\mu_{M}$ (see section 4).

\begin{tabular}{|c|c|c|c|c|c|}
\hline \multicolumn{6}{|c|}{ Summary of PDF Schemes and Default Parameters } \\
\hline PDF & Order & Scheme & $\mu_{M}$ & $m_{c}(\mathrm{GeV})$ & $m_{b}(\mathrm{GeV})$ \\
\hline CTEQ5F3 & $\alpha_{s}^{2}$ & FFNS & $Q$ & 1.3 & 4.5 \\
MRST2004FF3 & $\alpha_{s}^{2}$ & FFNS & $Q$ & 1.43 & 4.3 \\
CTEQ6.5 & $\alpha_{s}^{2}$ & VFNS & $\sqrt{Q^{2}+M^{2}}$ & 1.3 & 4.5 \\
MRST2004 & $\alpha_{s}^{2}$ & VFNS & $Q$ & 1.43 & 4.3 \\
MRST2004 NNLO & $\alpha_{s}^{3}$ & VFNS & $Q$ & 1.43 & 4.3 \\
\hline
\end{tabular}

Table 1: Summary of the theoretical schemes and default parameters used for comparison with the data in this paper.

A further consideration in the comparison of the heavy flavour PDFs with the HERA data is the data sets that were used as input in the analysis to extract the PDFs. The CTEQ and MRST PDFs are based mainly on the measurement of the inclusive neutral and charged current cross sections at HERA, inclusive fixed target data and additional constraints on the high $x$ gluon are obtained from jet measurements at the Tevatron. The CTEQ6.5 PDF also includes the HERA heavy flavour data sets $[1,2,4-6]$, and the MRST2004 PDFs include HERA $F_{2}^{c \bar{c}}$ data [3,4].

\section{Comparison with Experimental Data}

In this section the experimental data on inclusive charm and beauty production are compared with the PDFs from MRST and CTEQ introduced in section 2, A brief summary of the experimental methods employed to extract the data is also given. The comparison aims to highlight those areas where the theoretical predictions significantly differ.

\subsection{Inclusive Charm Production}

\subsubsection{Experimental Measurements}

Measurements of the inclusive charm structure function $F_{2}^{c \bar{c}}$ at HERA were first performed using the reconstructed decay products of $D$ mesons [3-6]. The measured visible cross sections of the $D$ meson are then extrapolated to the full phase space using theoretical models. At the lowest values of $Q^{2}$ the extrapolations can be large $(\sim 3-5[6])$ since the hadronic final state has a low probability to be in the restricted angular and transverse momentum ranges in which the measurements are made. In particular, experimental cuts on the transverse momentum of the $D$ meson are required to be high in order to suppress combinatorial background. The size of the extrapolation and, hence, the uncertainty on the extrapolation reduces with increasing $Q^{2}$ as the hadronic final state recoils with a larger transverse momentum. At large $Q^{2}\left(Q^{2}=500 \mathrm{GeV}^{2}\right)$ the extrapolation factor is much smaller in the range $\sim 1.6-2.4[6]$. The theoretical uncertainty 
on the extrapolation was investigated in [5] by using an alternative model based on CCFM evolution [26]. The differences were found to be as large as $20 \%$ at low $Q^{2}$ and $x$, reducing to around $5 \%$ at higher $Q^{2}$. In this paper the $D$ meson data shown from H1 and ZEUS are taken from the most recent publications $[5,6]$ based on the reconstruction of $D^{*}$ mesons. The experimental values of the charm structure function were calculated using the FFNS and the program HVQDIS to evaluate the extrapolations to the full phase space. In this procedure the predictions of HVQDIS for the differential $D^{*}$ meson cross sections are obtained by applying hadronisation corrections, estimated using Monte Carlo simulations and parameterisations of the $D^{*}$ fragmentation function [24], to the parton level predictions.

Recently measurements $[1,2]$ of the charm cross section have been made based on a technique which utilises the long lifetime of heavy flavour hadrons by measuring the displacement of tracks from the primary vertex. This allows access to lower momentum and a wider angular range than the reconstruction of exclusive charmed mesons. Provided the measurement is made in a range of $Q^{2}$ where the hadronic final state receives a large transverse momentum the technique can be used to measure the inclusive charm and beauty cross sections. The results from the displaced track and $D$ meson analyses are found to be very compatible [1,2]. It should be noted that although the extrapolation to the full phase space is reduced using the displaced track technique the combined statistical and systematic errors of the two methods, which arise from different sources, are of similar size.

\subsubsection{Comparison of Experimental Charm Data with PDFs}

Figure 1 shows the ratio of the charm structure function $F_{2}^{c \bar{c}}$ to the FFNS prediction of CTEQ5F3 as a function of $x$ for different values of $Q^{2}$ using the ZEUS $D^{*}$ binning scheme. The data from the $D^{*}$ meson and displaced track methods are shown as the points with errors. The H1 data points are interpolated to the nearest bin centre in $Q^{2}$ using a parameterisation of the cross section from a H1 FFNS NLO QCD fit as used in [2]. The $F_{2}^{c \bar{c}}$ measurements contain a small QCD correction from the effect of the longitudinal cross section. To avoid this correction in future the reduced cross section should be measured for heavy flavour production as was done in [2] and as has been the standard for the measurement of the inclusive DIS cross section for a longer time.

The data are compared with predictions using implementations of the NLO FFNS (CTEQ5F3, MRST2004FF3), the NLO VFNS (CTEQ6.5, MRST2004) and a NNLO VFNS (MRST2004 NNLO). Each of the three groups of predictions are discussed below.

The CTEQ FFNS PDF (CTEQ5F3) provides a good description of the data throughout the $Q^{2}$ range, in particular at low $Q^{2}$, supporting the hypothesis that the FFNS provides the correct description of heavy flavour production in this region. The MRST FFNS PDF (MRST2004FF3) is $50-30 \%$ lower than the CTEQ FFNS PDF at the lowest $Q^{2}$ value. This is a consequence of the matching of the FFNS partons to those of the VFNS at $Q^{2}=m_{c}^{2}$ and the MRST gluon distribution being smaller than CTEQ in the region of small $x$ and $Q^{2}$. The two FFNS PDFs show increasingly similar predictions with increasing $Q^{2}$ and are almost identical at $Q^{2}=$ $500 \mathrm{GeV}^{2}$. 
The two NLO VFNS PDFs (CTEQ6.5, MRST2004) show similar predictions of $F_{2}^{c \bar{c}}$ throughout the kinematic range of the figure. They both exhibit a shallower $x$ dependence and a different $Q^{2}$ evolution than the CTEQ FFNS PDF and are around 40\% lower than the CTEQ FFNS prediction at the lowest values of $Q^{2}$ and around $20 \%$ higher at the highest $Q^{2}$ values. In the low $Q^{2}$ region it may be expected that a VFNS PDF tends towards the FFNS result. This does not appear to be the case for the CTEQ PDFs which were extracted using different heavy quark factorization scales (see table 1) and different DIS data sets, as discussed in [8]. As stated above the MRST FFNS PDF is matched to the VFNS NLO PDF at low values of $Q^{2}$.

The matching of the MRST and FFNS partons at low $Q^{2}$ allows to investigate the difference in the evolution of the FFNS and VFNS partons with $Q^{2}$. The different evolution leads to a divergence, with the VFNS partons evolving more quickly than the FFNS partons, with increasing values of $Q^{2}$ with a convergence again at the highest $Q^{2}$. As discussed in [11] this is attributed to missing terms in the FFNS evolution. A similar behaviour was observed in an earlier comparison of FFNS and VFNS predictions for charm production [25].

The NNLO VFNS PDF (MRST2004 NNLO) improves considerably the description of the charm data at the lowest $Q^{2}$ values when compared with the MRST NLO VFNS PDF. For the rest of the phase space the NNLO VFNS prediction mostly lies between those of the MRST NLO VFNS and FFNS, only rising to the VFNS prediction at $Q^{2}=500 \mathrm{GeV}^{2}$.

For the low $Q^{2}$ and low $x$ charm data, the good description provided by the CTEQ NLO FF PDF compared with the need for higher orders to improve the description by MRST indicates that this region is sensitive to the differences in the gluon distribution, heavy flavour scheme and the order of $\alpha_{s}$ used in the calculations. For example, the ZEUS-JETS NLO PDF [18], which implements the Thorne-Roberts VFNS for the treatment of heavy flavours, gives predictions for charm (not shown) which lie between the MRST2004 and CTEQ5F3 PDFs for the three lowest $Q^{2}$ bins. The difference with respect to MRST2004 arises solely from the ZEUS-JETS gluon distribution being larger than MRST2004 in this region, whereas the difference to CTEQ5F3 is due to differences in both the scheme and gluon.

The data and theoretical predictions are compared again in figure 2 this time as a function of $Q^{2}$ for different values of $x$. The charm data show positive scaling violations for the majority of the measured values of $x$ with the slopes being steeper than the those for $F_{2}$ at the corresponding values of $x$, reflecting the dependence of the cross section on the gluon distribution of the proton. The features of the theoretical predictions discussed above are again evident. The description of the data is generally very good over the HERA kinematic range, with the CTEQ5F3 and MRST NNLO PDFs providing the best description in the region of low $x$ and $Q^{2}$.

\subsubsection{Summary of Inclusive Charm Production}

The theoretical description of the inclusive DIS charm cross section is seen to be generally very successful. A similar picture has been observed for the differential $D^{*}$ meson cross section data in both DIS $[5,6,27]$ and photoproduction $[28,29]$ with the failures of the theoretical description confined to particular corners of phase space. The good description of the charm data is likely to be a consequence of the fact that charm forms a significant contribution of the total cross section and is thus partly constrained by fits to the inclusive cross section. However, the increasing precision of the charm data themselves mean that the range of theoretical predictions is now comparable to the spread of the experimental data. 


\subsection{Beauty Production}

\subsubsection{Experimental Techniques}

The first measurement of beauty production in DIS was made by ZEUS using the relative transverse momentum of muons relative to jets $\left(p_{T}^{\mathrm{rel}}\right)$ [30]. The results were compared to NLO FFNS predictions using the parton level calculation from HVQDIS and hadronisation corrections estimated using Monte Carlo simulations. The ZEUS data were found to lie around 2.5 $\sigma$ above the theoretical predictions. An analysis by H1 [31], which combined the $p_{T}^{\mathrm{rel}}$ method with a precise measurement of the displacement of the muon track from the primary vertex using the silicon tracker, produced a similar conclusion and the data were found to lie $1.8 \sigma$ above the NLO predictions. The analysis technique based on the displacement of tracks due to the large lifetime of beauty hadrons allowed a measurement of the inclusive beauty DIS cross section at higher values of $Q^{2}[1,2]$. The predictions of QCD were found to be in good agreement with the data although the experimental errors are somewhat larger when compared with the muon based analyses.

\subsubsection{Comparison of Experimental Beauty Data with PDFs}

In figure 3 the ratio of the beauty structure function $F_{2}^{b \bar{b}}$ to the FFNS prediction of CTEQ5F3 as a function of $x$ for different values of $Q^{2}$ using the same binning as for the studies of $F_{2}^{c \bar{c}}$ is shown. The experimental data, obtained using the displaced track method [1,2], are shown as the points with error bars. The predictions for $F_{2}^{b \bar{b}}$ for the FFNS (CTEQ5F3, MRST2004FF3), the NLO VFNS (CTEQ6.5, MRST2004) and a NNLO VFNS (MRST2004 NNLO) are also shown and discussed below.

For the two FFNS PDFs the predictions for beauty production are very similar throughout the phase space, including the lowest values of $Q^{2}$, which is in contrast to the predictions for charm production. As will be shown in section 5 this is a consequence of the beauty predictions accessing the gluon at higher values of the gluon momentum fraction $x_{g}$ where the differences between the gluon distributions are smaller.

As was the case with the $F_{2}^{c \bar{c}}$ predictions the CTEQ VFNS PDF (CTEQ6.5) differs considerably from the CTEQ FFNS PDF at low $Q^{2}$. The difference is much larger for beauty than for charm with the VFNS prediction being $60-80 \%$ lower than the FFNS prediction for $Q^{2}$ values up to $18 \mathrm{GeV}^{2}$. The present experimental data at $Q^{2}=11 \mathrm{GeV}^{2}$ is compatible with both of the CTEQ predictions. The MRST VFNS PDF is higher than that from CTEQ for all values of $Q^{2}$, with the difference still around $20 \%$ at $Q^{2}=500 \mathrm{GeV}^{2}$.

As in the case of charm production the MRST FFNS and VFNS partons may be used to investigate the difference in evolution of the two schemes. The VFNS partons evolve quicker with increasing $Q^{2}$ than the FFNS partons with the divergence continuing over the kinematic range shown, resulting in a difference of $30 \%$ at $Q^{2}=500 \mathrm{GeV}^{2}$.

The NNLO VFNS PDF from MRST has a very different $x$ dependence and normalisation when compared with the NLO VFNS at the lowest $Q^{2}$ values. The NNLO PDF then evolves much quicker than the NLO PDF being $20 \%$ larger at $Q^{2}=18 \mathrm{GeV}^{2}$. This is followed by 
a much slower evolution such that the NNLO prediction is $20 \%$ below the NLO prediction at $Q^{2}=500 \mathrm{GeV}^{2}$.

It should be noted the predictions for inclusive beauty production using the ZM-VFNS are zero below $Q^{2}=m_{b}^{2} \sim 20 \mathrm{GeV}^{2}$. As can be seen in figure 3 the region of phase space below the 'mass threshold' is experimentally accessible. The measurements of beauty production significant from zero at $Q^{2}=11 \mathrm{GeV}^{2}$ highlight the fact that the ZM-VFNS is inapplicable for the description of heavy flavour production in the kinematic range of HERA.

\subsubsection{Summary of Inclusive Beauty Production}

The inclusive beauty data are well described by all of the theoretical predictions, within the large statistical errors, with no indication of the excess over theory as observed in the final state measurements. The differences between any set of theoretical predictions are generally smaller for beauty production than they are for charm due to the region of $x_{g} g(x)$ probed. However, in the region $Q^{2}<m_{b}^{2}$ there are much larger differences between the theoretical predictions. One possibility for the large spread in the predictions in this region is that beauty is a small contribution to the total cross section and the experimental uncertainties on the beauty cross section measurements are still large. The large beauty mass means that, in contrast to charm,

$Q^{2}$ values well below $Q^{2}=M^{2}$ but still in a region where pQCD may be expected to work are experimentally accessible.

\section{Theoretical Uncertainties}

The theoretical uncertainty due to the effects of missing higher order corrections on QCD predictions performed at a fixed order is usually estimated by varying the factorization and renormalization scales in the calculation. The precise prescription adopted varies considerably. This is particularly true for heavy flavours where there are further degrees of freedom, in comparison to the total inclusive cross section, due to the mass of the heavy quarks. It should be noted that in kinematic regions where the chosen QCD scheme is a poor approximation the variation of the perturbative scales does not provide an estimate of the effect of the neglected contributions. An illustrative example of this is the ZM-VFNS for values of $Q^{2}$ below the heavy flavour 'threshold' $Q^{2}=M^{2}$ where the cross section prediction is zero and the variation of the perturbative scale has no effect.

In this section a comparison of the size of the theoretical uncertainty on inclusive charm and beauty cross sections is investigated using the FFNS (CTEQ5F3) as an example. In figure 4 the uncertainty on $F_{2}^{c \bar{c}}$ and $F_{2}^{b \bar{b}}$ due to the variation of the factorization and renormalization scales is shown. The default scales are chosen as $\mu_{f}=\mu_{r}=Q$.

In the figure the variation of the factorization scale $\mu_{f}$ from $Q$ to $2 Q$ leads to large increases in the predictions, particularly at low $Q^{2}$. The uncertainty due to varying $\mu_{f}$ for charm is significantly larger than for beauty at the lowest values of $Q^{2}$, and arises from the difference in the region of $x_{g}$ probed (see section 5). The variation of the factorization scale from $Q$ to $Q / 2$ (not shown) leads to decreases in the predictions of similar magnitude. 
The choice of $Q$ as the renormalization scale means that the variation of $\mu_{r}$ from $Q$ to $2 Q(Q / 2)$ leads to similar effects for charm and beauty (not shown). The uncertainty due to these changes is extremely large at the lowest value of $Q^{2}-50 \%(+500 \%)$ and decreases with increasing $Q^{2}$ to around $-20 \%(+30 \%)$ for the highest $Q^{2}$ value shown. The variations reflect the behaviour of the strong coupling $\alpha_{s}\left(\mu_{r}\right)$ with the scale $\mu_{r}$.

For predictions of final state cross sections larger perturbative scales than $Q^{2}$ are often used, typically $\mu_{f}=\mu_{r}=\sqrt{Q^{2}+4 M^{2}}$ [23], which provide a better estimation of the virtuality of the hard scattering process. The relative changes to the predictions for the scale choice $\mu_{f}=\mu_{r}=\sqrt{Q^{2}+4 M^{2}}$ are also shown in the figure. Due to the large mass of the beauty quark there is a large suppression of the cross section, particularly at low $Q^{2}$. The effect is much smaller for charm production. For the scale choice $\mu_{f}=\mu_{r}=\sqrt{Q^{2}+4 M^{2}}$ the variation of $\mu_{f}$ and $\mu_{r}$ by factors 2 and $1 / 2$ leads to improved stability in the predictions than those observed for the scale choice $\mu_{f}=\mu_{r}=Q$. For example, the variation of $\mu_{r}$ by 0.5 leads to maximum changes of $+70 \%(+30 \%)$ for charm (beauty) and the variation of $\mu_{f}$ by factors 0.5 and 2 leads to maximum changes of $\pm 40 \%$ for charm and $\pm 20 \%$ for beauty.

At higher values of $Q^{2}$, where the role of the heavy quark masses in the perturbative scale becomes decreasingly important, the uncertainty on the charm and beauty cross sections becomes similar. At values of $Q^{2}=500 \mathrm{GeV}^{2}$ the combined theoretical uncertainty is around $20 \%$. This is to be compared with the differences between the predictions of the MRST FFNS and VFNS partons of $\sim 20 \%$ for charm and $\sim 30 \%$ for beauty at $Q^{2}=500 \mathrm{GeV}^{2}$.

In summary the uncertainty due to variations of the scale are large, particularly at low $Q^{2}$. The large uncertainty from variation of the scales when using $\mu_{f}=\mu_{r}=Q$ is reduced by using the larger scale $\mu_{f}=\mu_{r}=\sqrt{Q^{2}+4 M^{2}}$. The fact that charm production gives a significant contribution to the total cross section suggests that the scale uncertainty may be overestimated in this manner i.e. the inclusive DIS cross section will be poorly described with the changed charm scale. To compensate for this effect, the PDFs given in [32] based on CCFM evolution, are available for a number of different choices of scale for the heavy flavour contribution, which reduces the scale uncertainty when evaluated as in the manner above.

\section{Comparison of the CTEQ and MRST Gluon Distributions}

In this section the differences in the CTEQ and MRST gluon distributions at low $Q^{2}$ and $x$ are highlighted since they have an important effect on the predictions for the inclusive charm cross section. At leading order the cross section for heavy flavour production in the FFNS is given by the convolution $\sigma \propto \int_{a x}^{1} C\left(x / x_{g}, \mu^{2}\right) g\left(x_{g}\right) d x_{g} / x_{g}$ where $C\left(x / x_{g}, \mu^{2}\right)$ is the coefficient function and $g\left(x_{g}\right)$ is the gluon distribution. The integral is calculated over the range from threshold values of $x_{g}=a x$ where $a=1+4 M^{2} / Q^{2}$ up to a maximum value of $x_{g}=1$. The coefficient functions have the feature that the average value of $x_{g}$ is weighted away from $x=x_{g}$ and the convolution results in probing the gluon distribution at higher values of $x_{g}$. Therefore, at low values of $Q^{2}$ the minimum value of $x_{g}$ probed $\left(x_{g}^{\min }=a x\right)$ is higher for beauty production than charm. This is illustrated in figure 5 where the CTEQ and MRST FFNS

\footnotetext{
${ }^{4}$ At NLO the weighting away from $x=x_{g}$ in the coefficient function increases.
} 
gluon distributions are compared as a function of $x_{g}$ at different values of $\mu^{2}=Q^{2}$. For each bin the minimum $x_{g}$ value of the experimental $F_{2}^{c \bar{c}}$ charm data is indicated as well as the minimum $x_{g}$ value for charm and beauty production. As can be seen in the figure, the charm data is more sensitive to the large difference in the CTEQ and MRST FFNS gluon distributions at low $Q^{2}$ and $x_{g}$. The differences in the gluon distributions contribute to the differences in the charm cross section between CTEQ5F3 and MRST2004FF3 seen in the lowest $Q^{2}$ regions of figure 1 . The difference in the charm cross section for the two PDFs is smaller than the difference in the gluon PDFs because of the convolution of the gluon distribution with the heavy flavour coefficients. The similarity of the CTEQ and MRST FFNS gluon distributions for the $x_{g}$ range sensitive to beauty production explains why the large differences at low $Q^{2}$ between CTEQ5F3 and MRST2004FF3 observed for $F_{2}^{c \bar{c}}$ are not evident for $F_{2}^{b \bar{b}}$ (figure 3).

\section{Summary and Outlook}

In this paper the latest theoretical predictions for heavy flavour production in DIS have been introduced and the status of the comparison with the experimental data from HERA investigated. The inclusive charm data, which form a sizeable fraction of the total cross section, are seen to be generally well described by the predictions. However, the increasing precision of the charm data mean that the range of theoretical predictions is now comparable to the spread of the experimental data. The production of charm is particularly sensitive to the gluon distribution of the proton. Future measurements of the inclusive charm cross section from the HERA-II data will continue to provide tests of the theoretical models and PDFs.

The inclusive production of beauty is also well described by the QCD predictions. The production of beauty in the region $Q^{2}<m_{b}^{2} \sim 20 \mathrm{GeV}^{2}$ is particularly sensitive to the scheme and perturbative scale chosen. The theoretical understanding will clearly benefit from the increased statistics in this region that will become available from the HERA II data.

The theoretical predictions for charm and beauty show sensitivity in the $Q^{2}$ dependence due to different evolution schemes, although the present theoretical picture is complicated by mass threshold and other effects. The sensitivity of heavy flavour production to the parton distributions of the proton will continue to improve the reliability of QCD predictions of standard model and new physics processes at the Tevatron and LHC.

\section{Acknowledgements}

I am grateful to R. S. Thorne and W. K. Tung for providing me with their calculations and for productive discussions.

\section{References}

[1] A. Aktas et al. [H1 Collaboration], Eur. Phys. J. C 40 (2005) 349 [hep-ex/0411046]. 
[2] A. Aktas et al. [H1 Collaboration], Eur. Phys. J. C 45 (2006) 23 [hep-ex/0507081].

[3] C. Adloff et al. [H1 Collaboration], Z. Phys. C 72 (1996) 593 [hep-ex/9607012].

[4] J. Breitweg et al. [ZEUS Collaboration], Eur. Phys. J. C 12 (2000) 35 [hep-ex/9908012].

[5] C. Adloff et al. [H1 Collaboration], Phys. Lett. B 528 (2002) 199 [hep-ex/0108039].

[6] S. Chekanov et al. [ZEUS Collaboration], Phys. Rev. D 69 (2004) 012004 [hep-ex/0308068].

[7] H. L. Lai et al. [CTEQ Collaboration], Eur. Phys. J. C 12 (2000) 375 [hep-ph/9903282].

[8] W. K. Tung, H. L. Lai, A. Belyaev, J. Pumplin, D. Stump and C. P. Yuan, [hep-ph/0611254].

[9] A. D. Martin, R. G. Roberts, W. J. Stirling and R. S. Thorne, Eur. Phys. J. C 39 (2005) 155 [hep-ph/0411040].

[10] R. S. Thorne, Phys. Rev. D 73 (2006) 054019 [hep-ph/0601245].

[11] A. D. Martin, W. J. Stirling and R. S. Thorne, Phys. Lett. B 636 (2006) 259 [hep-ph/0603143].

[12] E. Laenen, S. Riemersma, J. Smith and W. L. van Neerven, Nucl. Phys. B 392 (1993) 162;

E. Laenen, S. Riemersma, J. Smith and W. L. van Neerven, Nucl. Phys. B 392 (1993) 229.

[13] B. W. Harris and J. Smith, Nucl. Phys. B 452 (1995) 109 [hep-ph/9503484].

[14] C. Adloff et al. [H1 Collaboration], Eur. Phys. J. C 21 (2001) 33 [hep-ex/0012053].

[15] S. Chekanov et al. [ZEUS Collaboration], Phys. Rev. D 67 (2003) 012007 [hep-ex/0208023].

[16] A. Aktas et al. [H1 Collaboration], [hep-ex/0610076].

[17] R. S. Thorne and R. G. Roberts, Phys. Rev. D 57 (1998) 6871 [hep-ph/9709442];

R. S. Thorne and R. G. Roberts, Phys. Lett. B 421 (1998) 303 [hep-ph/9711223];

R. S. Thorne and R. G. Roberts, Eur. Phys. J. C 19 (2001) 339 [hep-ph/0010344].

[18] S. Chekanov et al. [ZEUS Collaboration], Eur. Phys. J. C 42 (2005) 1 [hep-ph/0503274].

[19] J. C. Collins and W. K. Tung, Nucl. Phys. B 278 (1986) 934.

M. A. G. Aivazis, F. I. Olness and W. K. Tung, Phys. Rev. D 50 (1994) 3085 [hep-ph/9312318];

M. A. G. Aivazis, J. C. Collins, F. I. Olness and W. K. Tung, Phys. Rev. D 50 (1994) 3102 [hep-ph/9312319];

J. C. Collins, Phys. Rev. D 58 (1998) 094002 [hep-ph/9806259].

[20] W. K. Tung, S. Kretzer and C. Schmidt, J. Phys. G 28 (2002) 983 [hep-ph/0110247]. 
[21] M. Krämer, F. I. Olness and D. E. Soper, Phys. Rev. D 62 (2000) 096007 [hep-ph/0003035].

[22] W. M. Yao et al. [Particle Data Group], J. Phys. G 33 (2006) 1.

[23] S. Alekhin et al., "HERA and the LHC - A workshop on the implications of HERA for LHC physics: Proceedings Part B," p 405. [hep-ph/0601013].

[24] C. Peterson, D. Schlatter, I. Schmitt and P.M. Zerwas, Phys. Rev. D 27 (1983) 105.

[25] A. Chuvakin, J. Smith and B. W. Harris, Eur. Phys. J. C 18 (2001) 547 [hep-ph/0010350].

[26] M. Ciafaloni, Nucl. Phys. B 296 (1988) 49;

S. Catani, F. Fiorani and G. Marchesini, Phys. Lett. B 234 (1990) 339;

S. Catani, F. Fiorani and G. Marchesini, Nucl. Phys. B 336 (1990) 18;

G. Marchesini, Nucl. Phys. B 445 (1995) 49 [hep-ph/9412327].

[27] A. Aktas et al. [H1 Collaboration], [hep-ex/0701023].

[28] J. Breitweg et al. [ZEUS Collaboration], Eur. Phys. J. C 6 (1999) 67 [hep-ex/9807008];

S. Chekanov et al. [ZEUS Collaboration], Phys. Lett. B 565 (2003) 87 [hep-ex/0302025];

S. Chekanov et al. [ZEUS Collaboration], Nucl. Phys. B 729 (2005) 492 [hep-ex/0507089].

[29] A. Aktas et al. [H1 Collaboration], [hep-ex/0608042].

[30] S. Chekanov et al. [ZEUS Collaboration], Phys. Lett. B 599 (2004) 173 [hep-ex/0405069].

[31] A. Aktas et al. [H1 Collaboration], Eur. Phys. J. C 41 (2005) 453 [hep-ex/0502010].

[32] H. Jung, [hep-ph/0411287]. 


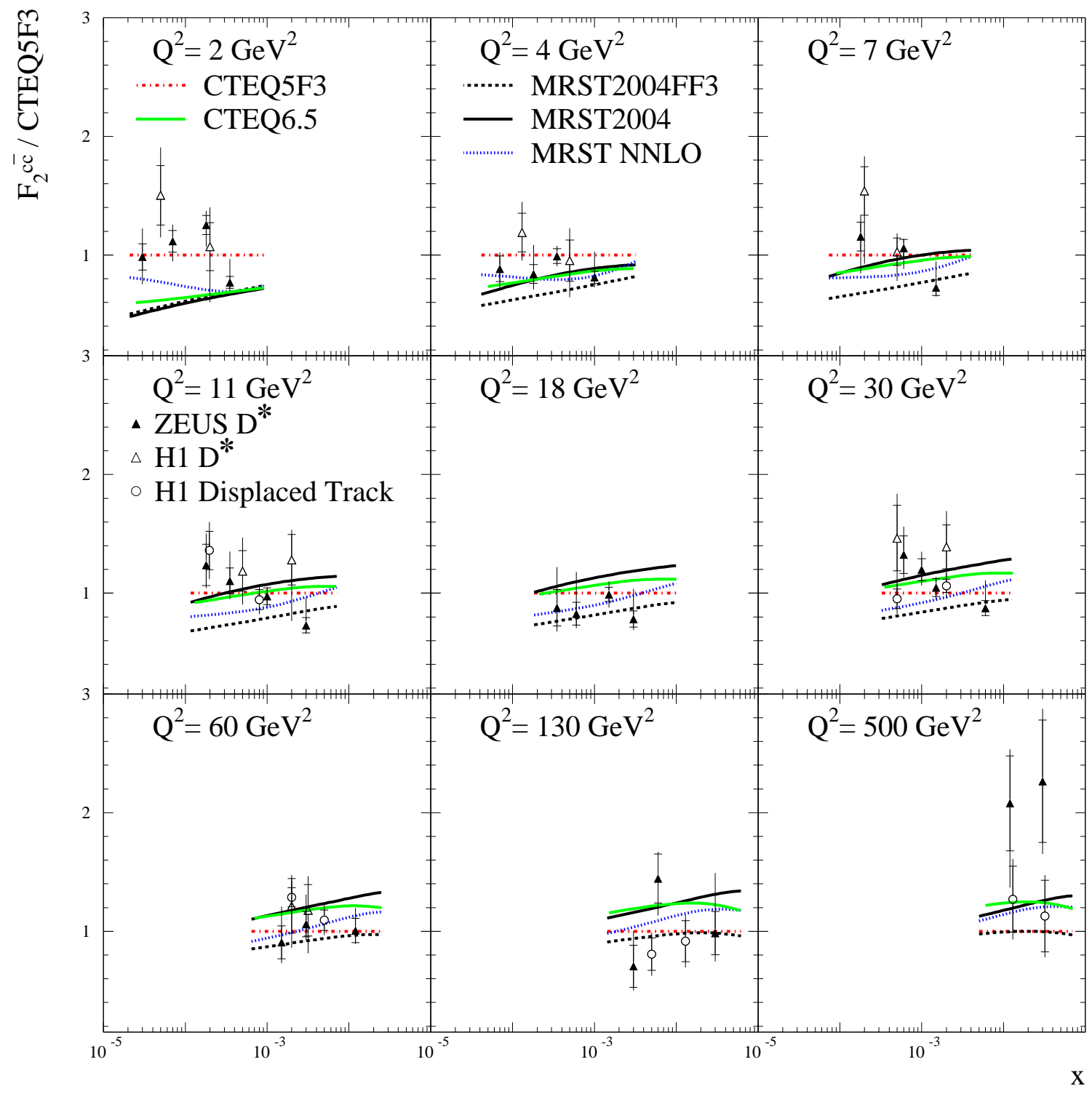

Figure 1: The ratio of the charm structure function $F_{2}^{c \bar{c}}$ to the prediction of CTEQ5F3 shown as a function of $x$ for different values of $Q^{2}$. The data obtained using reconstructed $D^{*}$ mesons and the displacement of tracks from the primary vertex are shown as points. The inner error bar corresponds to the statistical uncertainty and the outer error bar to the statistical and systematic errors added in quadrature. The data are compared with the theoretical predictions of perturbative QCD using different prescriptions for the heavy flavour treatment from the MRST and CTEQ fitting groups. 


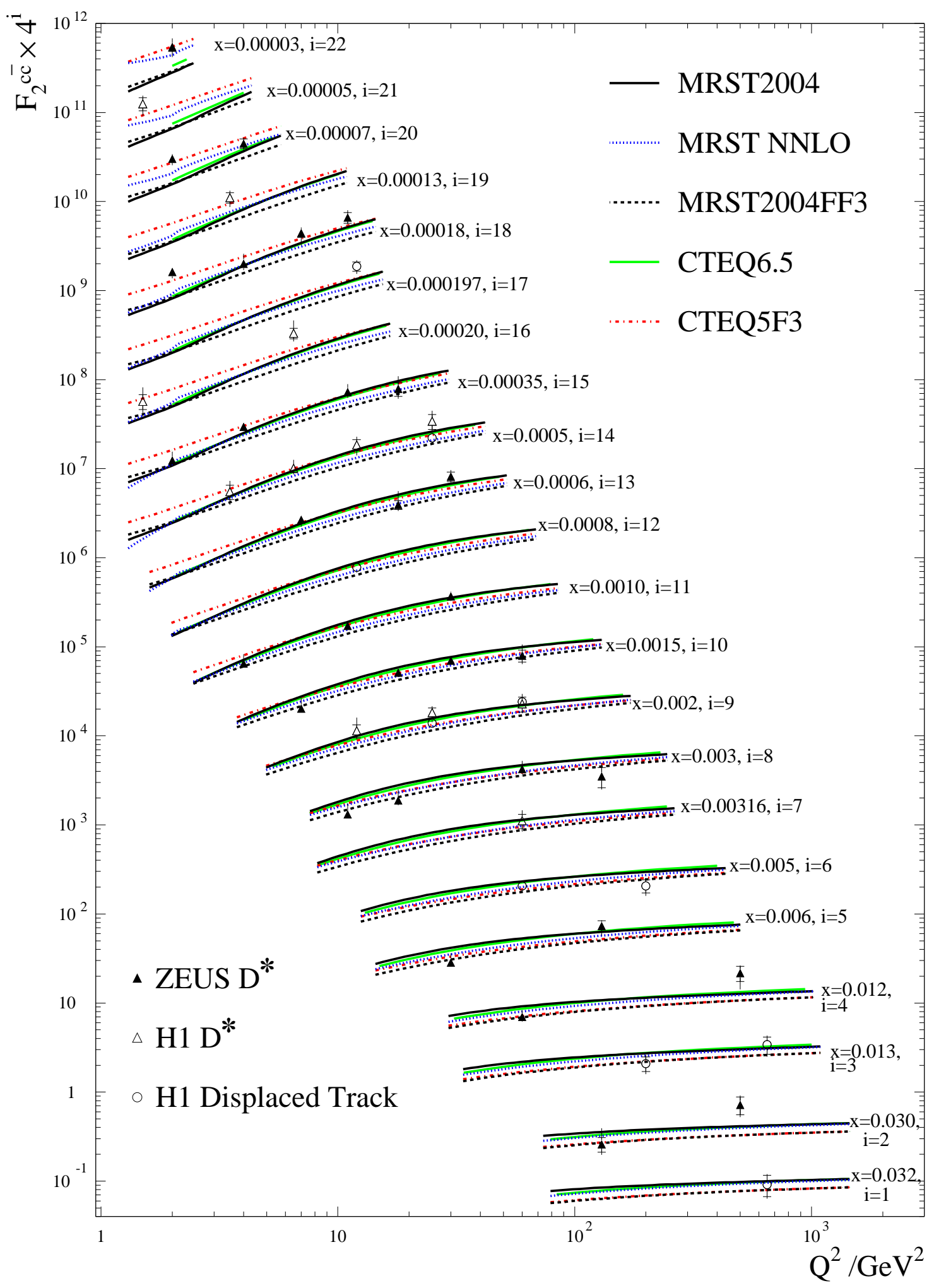

Figure 2: The charm structure function $F_{2}^{c \bar{c}}$ as a function of $Q^{2}$ for different values of $x$. The data obtained using reconstructed $D^{*}$ mesons and the displacement of tracks from the primary vertex are shown as points. The data are compared with the theoretical predictions of perturbative QCD using different prescriptions for the heavy flavour treatment from the MRST and CTEQ fitting groups. 


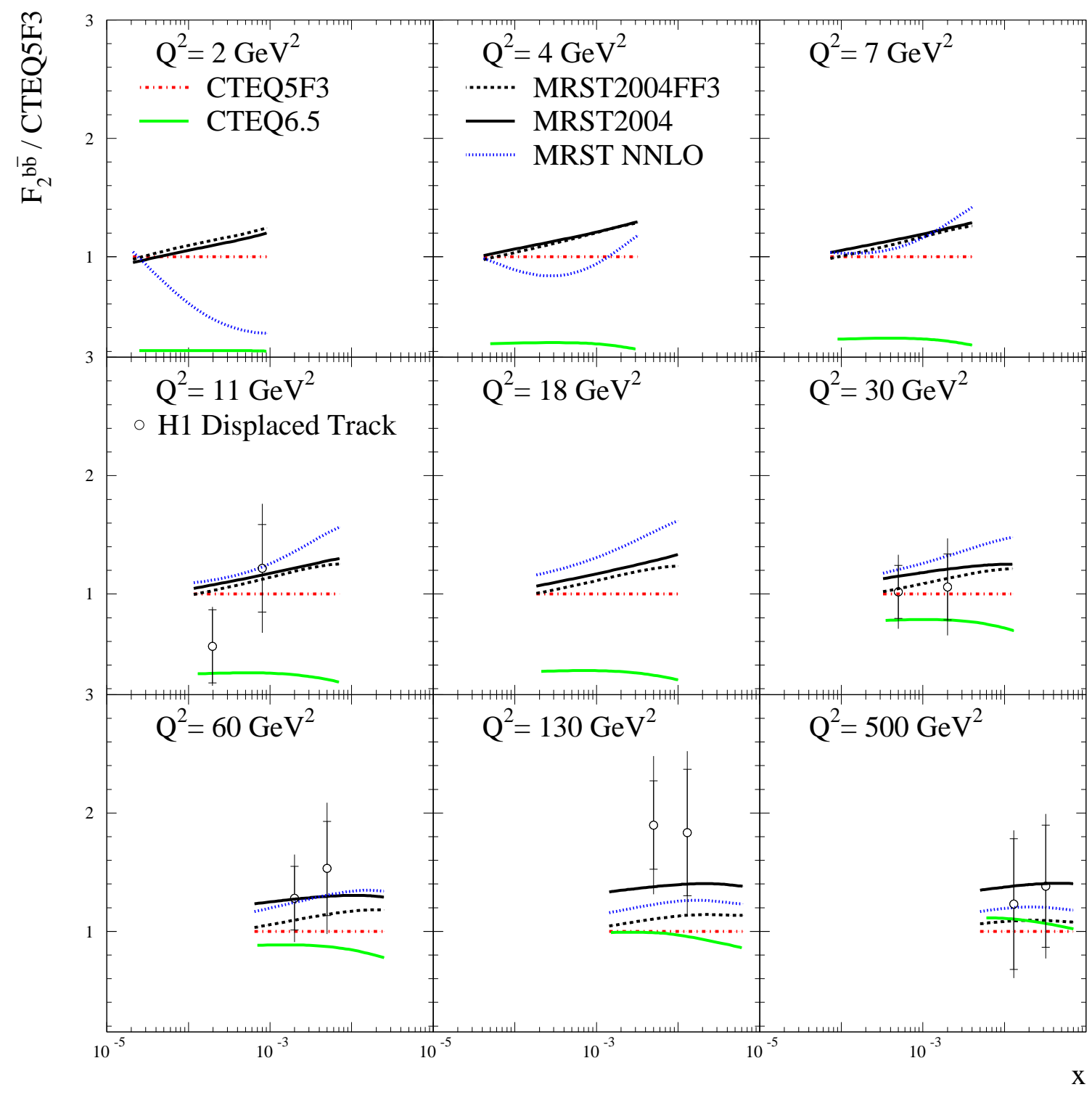

Figure 3: The ratio of the beauty structure function $F_{2}^{b \bar{b}}$ to the prediction of CTEQ5F3 as a function of $x$ for different values of $Q^{2}$. The data obtained using the displacement of tracks from the primary vertex are shown as points. The inner error bar corresponds to the statistical uncertainty and the outer error bar to the statistical and systematic errors added in quadrature. The data are compared with the theoretical predictions of perturbative QCD using different prescriptions for the heavy flavour treatment from the MRST and CTEQ fitting groups. 


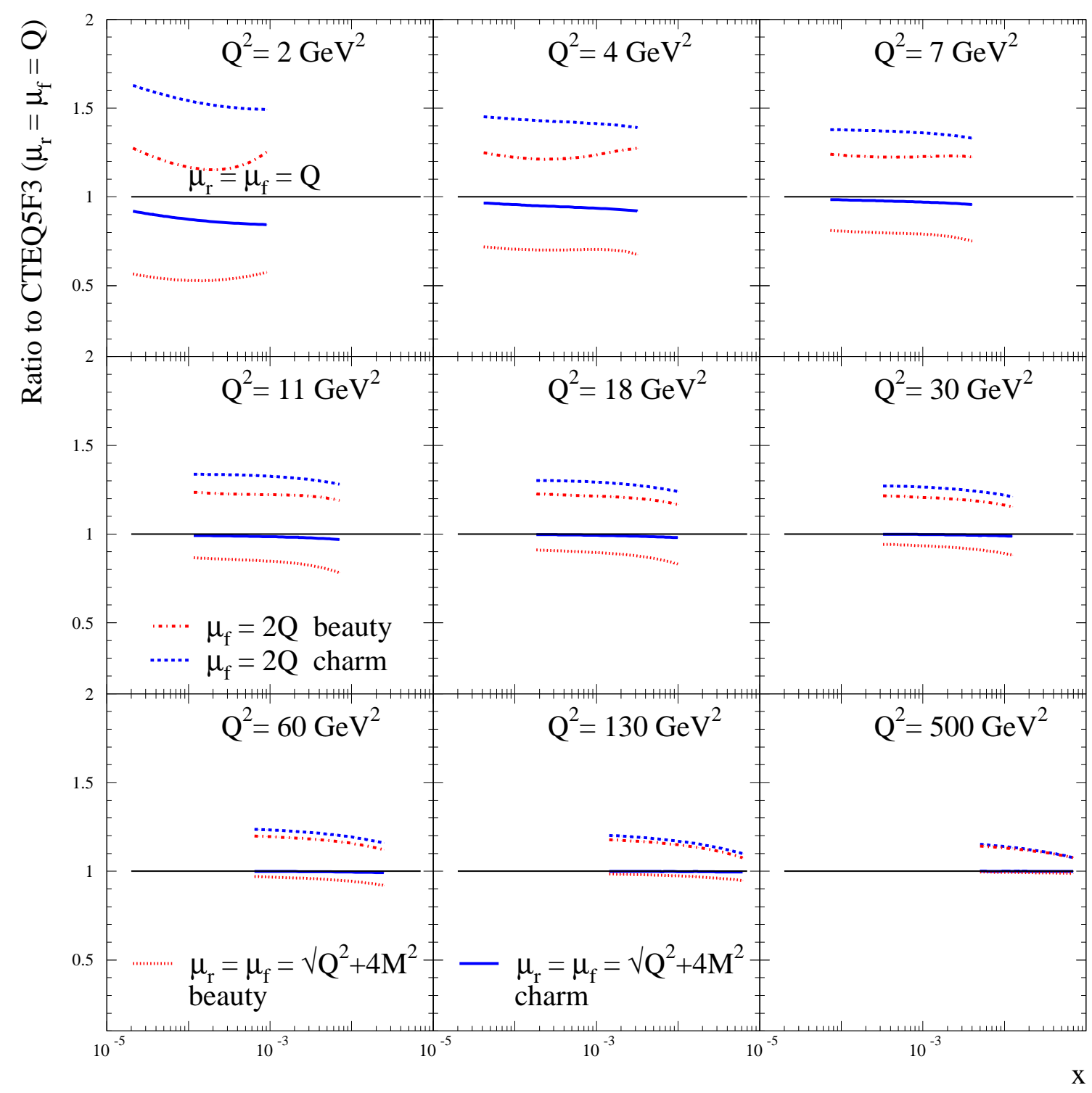

Figure 4: The effect of varying the renormalization and factorization scales for the inclusive charm and beauty DIS cross section shown as a function of $x$ for different values of $Q^{2}$. The predictions are shown using the CTEQ5F3 PDF with the default scale $\mu_{r}=\mu_{f}=Q$ and alternatively with $\mu_{r}=\mu_{f} / 2=Q$ and $\mu_{r}=\mu_{f}=\sqrt{Q^{2}+M^{2}}$. 


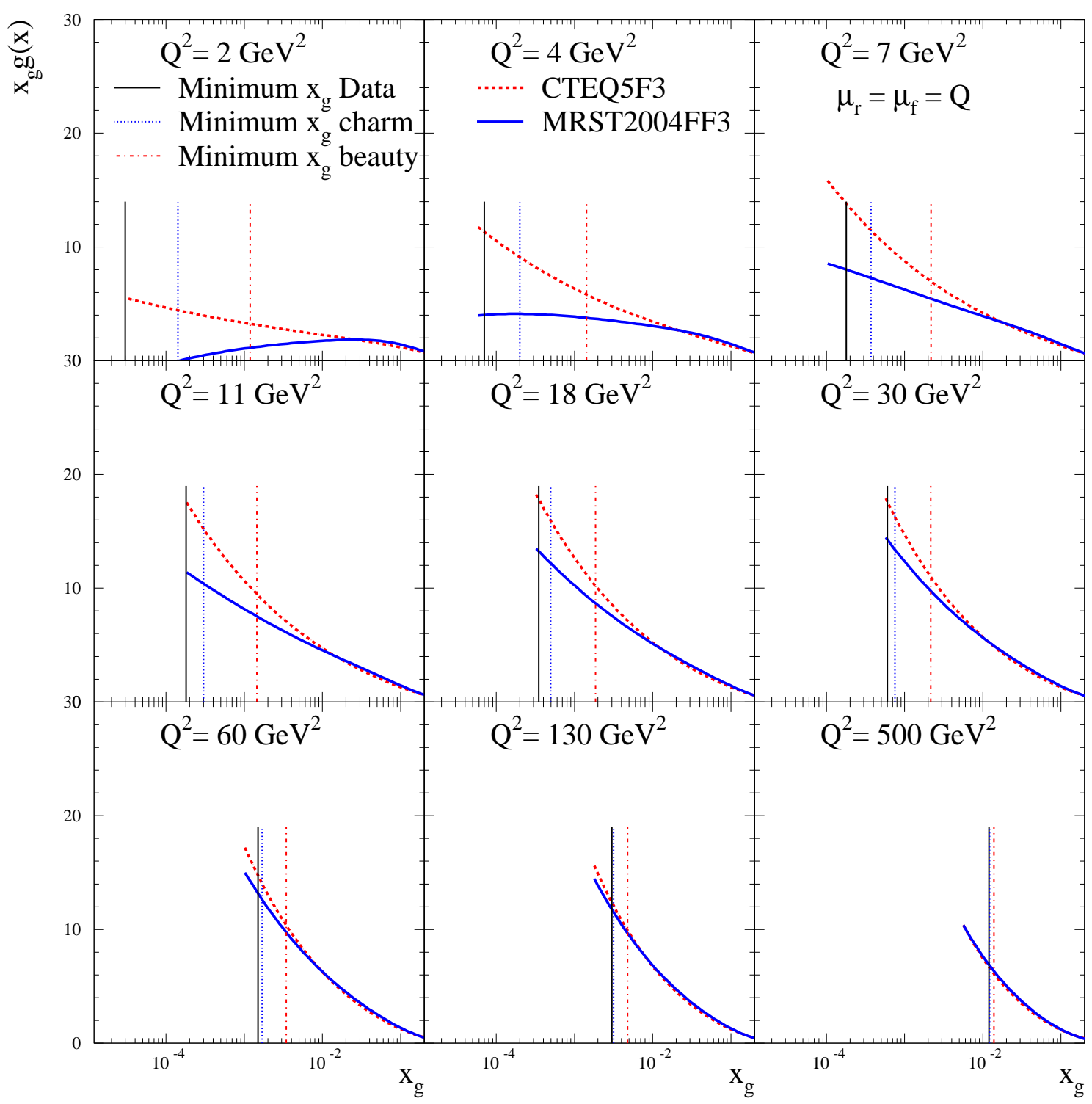

Figure 5: The CTEQ and MRST gluon distributions shown as a function of $x_{g}$ for different values of $\mu^{2}=Q^{2}$. The vertical solid lines indicate the range of experimental $F_{2}^{c \bar{c}}$ data. The vertical dashed and, dashed-dotted lines indicate the effective range probed for charm and beauty production, respectively. 